\title{
Virtual Scene Velocity Influences Postural Responses to an Inclined Base of Support
}

\author{
Yun Wang, Robert V. Kenyon and Emily A. Keshner
}

\begin{abstract}
Orientation of the body has been shown to adapt to sustained stance on a tilted support surface in subjects who were blindfolded or who had their eyes closed. Postural adaptation occurs even when the lower limbs are constrained, suggesting that there is central adaptation of the perception of vertical. These studies, however, were performed in the absence of visual inputs and it is not clear whether the presence of visual inputs would alter a central adaptive response to a physical displacement in space. In this study we have explored the relative contributions of surface tilt and visual velocity on the initial process of realignment to the vertical. Pitch motion of the visual field was presented at varying velocities to eight subjects $(29.9 \pm \mathbf{2 . 8}$ yrs $)$ standing quietly on a 3 deg toes-up tilt of the base of support. Onset of virtual scene and platform movement were synchronized in all trials. We hypothesized that motion of the visual world would affect the ability to return to the initial vertical position while standing on a tilted base of support. We found that in the first few seconds following stance on a tilted base of support, orientation in space was greatly influenced by the velocity of visual scene motion.
\end{abstract}

\section{INTRODUCTION}

$\mathrm{R}$ ECENT studies [1]-[3] have demonstrated that standing on a tilted surface for several minutes when vision was absent produced temporary aftereffects so that subjects continued to lean even after the tilt had been removed. The postural after-effect occurred whether the subject was standing or stepped on the tilted surface. Constraint of the joints and muscles that were most affected by the tilted surface (i.e. ankles and lower body musculature) did not keep the trunk from leaning which suggested that there was a process of central adaptation of a surface-referenced set point [1], [2].

The central set-point for postural orientation has been proposed to reside in several sites of the CNS. Some have proposed an internal model in the vestibular system which controls orientation in space in both animals [4] and humans

Manuscript received April 11, 2008. This work was supported by grants DC05235 from NIH-NIDCD and AG26470 from NIH-NIA.

Yun Wang was with the Sensory Motor Performance Program, Rehabilitation Institute of Chicago, Chicago IL 60611. She is now with the Department of Health and Sports Science, Graduate School of Education, Hiroshima University, Kagamiyama 1-1-1, Higashi-Hiroshima 739-8524, Japan (e-mail: yunwang@hiroshima-u.ac.jp).

Robert V. Kenyon is with Department of Computer Science, University of Illinois at Chicago, 1120 SEO Bldg (M/C 152), 851 S. Morgan St., Chicago, IL 60607, USA (e-mail: kenyon@uic.edu).

Emily A. Keshner was with the Sensory Motor Performance Program, Rehabilitation Institute of Chicago, Chicago IL 60611. She is now with the Department of Physical Therapy, College of Health Professions and Department of Electrical and Computer Engineering, College of Engineering, Temple University, 3307 N. Broad St, Jones Hall Room 600 Philadelphia, PA 19140, USA. (corresponding author phone: 215-707-4824; fax: 215-707-7500; e-mail: ekeshner@temple.edu).
[5]. The Mergner group [6]-[8] has posited that the vestibular signal supplies the global spatial orientation signal after subtracting out segmental proprioceptive feedback. The residual signal would then be compared to the internal model to determine orientation in space. It has also been proposed [9] that the internal model is formed using multiple sensory inputs and that only afferent changes of considerable duration would alter the internal representation of verticality.

There is, however, evidence that suggests that even short term disturbances may briefly alter the perception of postural vertical. Healthy subjects exhibited significant effects of visual field velocity on peak angular velocities of the head, but it was not determined if their ability to return to the upright position was affected [10]. In vestibular deficient individuals, the ability to return to a vertical position was observed to degrade gradually over the course of several trials of dorsiflexion tilt and eventually produced a backwards fall [11]. Thus, with vestibular loss or dysfunction, a delay in the comparison of afferent feedback with the internal model may have increased dependence upon the immediate sensory array to define vertical.

In this experiment we have explored the initial process of realignment to the vertical posture (i.e., aligned with their initial position) on a tilted support surface to identify which inputs are influencing the phasic perception of vertical. Specifically, we have provided pitch motion of the visual field at varying velocities while imposing an upward tilt of the base of support. We hypothesized that motion of the visual world would affect the ability to return to the initial vertical position when the gravitational reference from the base of support was not matched to the gravitational vertical. Our results support this hypothesis, and demonstrate that wide field of view motion affects the vertical orientation of the body in space differentially when standing on an earthreferenced base of support or following the automatic reaction to a tilted base of support. These results may be a step toward understanding the instability observed in patients with vestibular deficit when in visually demanding environments [12].

\section{METHODS}

\section{A. Subjects}

Eight ( 5 male, 3 female) healthy young adults $(29.9 \pm 2.8$ yrs) who were naïve to virtual environments participated in this study. All were free of any known musculoskeletal or neurological disorders. All subjects provided written consent in accordance with the Institutional Review Board, Feinberg School of Medicine, Northwestern University. 


\section{B. Apparatus}

The platform was placed within a single-wall virtual reality system (Fig. 1). The screen in our system consisted of back projection material measuring $2.6 \mathrm{~m}$ x $3.2 \mathrm{~m}$. An Electrohome Marquis 8500 projector threw a full-color stereo workstation field $(1024 \times 768$ stereo $)$ at $120 \mathrm{~Hz}$ onto the screen that filled the horizontal and vertical field of view. A dual processor PC with a nVidia Quadro [FX3000G] graphics card created the imagery projected onto the wall using the CAVELib (VRCO, Inc) VR library. The field sequential stereo images generated by the PC were separated into right and left eye images using liquid crystal stereo shutter glasses worn by the subject (Crystal Eyes, StereoGraphics Inc.) which limited the horizontal and vertical field of view to $100^{\circ}$ and $55^{\circ}$ of binocular vision. The projected image (scene) consisted of a $30.5 \mathrm{~m}$ wide by $6.1 \mathrm{~m}$ high by $30.5 \mathrm{~m}$ deep room containing round columns with patterned rugs and a painted ceiling.

Subjects stood upon a platform (Neurocom Inc. Clakamas, OR, USA) that either remained stationary or rotated in the sagittal plane ( $3^{\circ}$ of dorsiflexion) with a constant velocity ramp of $30 \%$ sec. A six camera Motion Analysis (Motion Analysis, Inc., Santa Rosa, CA) system was used to capture joint motion at $120 \mathrm{~Hz}$. Infrared markers were attached bilaterally on the second metatarsophalangeal joint, lateral malleolus, lateral epicondyle of the tibia, greater trochanter of the femur, acromion process, lateral epicondyle of the humerus, styloid process of the ulna, second metacarpophalangeal joint, zygomatic arch, and the external auditory meatus of the ear. Markers were also placed on C7 and L4-L5 of the spine and on the occipital region of the head.

\section{Procedures}

Subjects stood comfortably on the platform with their feet side-by-side at hip width, and with their upper arms at their sides and bent at the elbows (Fig. 1). Foot position was marked on the platform and reproduced across trials. Subjects were asked to maintain an erect posture while standing in the dark or in front of a virtual scene with natural visual motion.

The virtual scene was externally driven at one of four constant velocities in the pitch up direction: $0 \% \mathrm{~s}$ (visible but stationary relative to head motion), $30 \% \mathrm{~s}, 45 \%$, and $60 \% \mathrm{~s}$. All trials lasted $65 \mathrm{sec}$ following onset of the virtual scene or platform tilt. Trials in which the platform was inclined consisted of a pre-incline period of $5 \mathrm{sec}$ when the support surface was held horizontal, an incline period of $30 \mathrm{sec}$ when the surface was maintained in the toe-up position following the $30 \% \mathrm{~s} \mathrm{ramp}$, and a post-incline period of $30 \mathrm{sec}$ when the surface slowly returned to the horizontal at a constant velocity of $0.1 \%$ s. In this paper we present data only for a 2.5 sec period preceding and following platform tilt, and for the same period of time with the stationary platform. Onset of virtual scene and platform movement were synchronized in all trials. The first $1.2 \mathrm{~s}$ following stimulus onset were excluded from the computation to allow the initial automatic postural reactions to subside and restabilization to a vertical orientation to be in progress [11]. Following each trial, there was a rest period with eyes closed for approximately one or two minutes. A total of 30 trials were presented in a pseudorandom order that included 3 trials of each of the five visual conditions and the two platform (stationary and tilted) conditions.

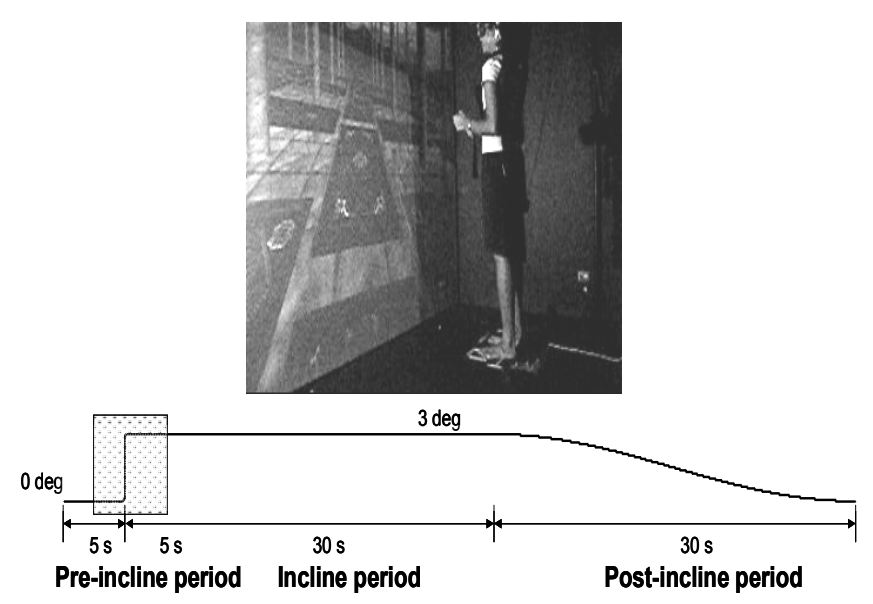

Fig. 1. Virtual environment displayed to the subjects standing on the platform (upper panel). The schema of the experimental setup is shown in the bottom panel: all trials lasted $65 \mathrm{sec}$ following stimulus (platform and scene) onset. Trials in which the platform was inclined consisted of a preincline period of $5 \mathrm{sec}$ when the support surface was held horizontal, an incline period of $30 \mathrm{sec}$ when the surface was maintained in the toe-up position following the $30 \% \mathrm{~s}$ ramp, and a post-incline period of $30 \mathrm{sec}$ when the surface slowly returned to the horizontal at a constant velocity of $0.1 \%$

\section{Data Processing}

A weighted average of the COM of each body segment [13] was calculated. A line drawn between markers on the external auditory meatus of the ear and the occiput was used to calculate head angular position relative to gravitational vertical. Ankle joint position was the angle between the $2^{\text {nd }}$ metatarsophalangeal joint and the lateral malleolus to the lateral malleolus and lateral epicondyle of the tibia in the sagittal plane. All data were low-pass filtered using a $4^{\text {th }}$ order Butterworth digital filter at $4 \mathrm{~Hz}$.

Mean position of whole body COM and angular displacements of the head and ankle were calculated. When visual scene motion was combined with a toe-up platform, latencies were computed at the time at which each response reached its peak. Comparisons across visual scene conditions were performed with a one-way ANOVA and Bonferroni post hoc comparisons at $p<0.05$. Comparisons between the two platform movements (stable and toe-up) were made with a Wilcoxon $t$-test for each visual scene condition.

\section{RESULTS}

\section{A. Whole body COM displacement}

When the platform was stable and the visual scene was moving, subjects exhibited forward motion of the COM (Fig. 2, top panel) which increased in amplitude in the pitch up direction as the velocity of the visual scene increased. There was a significant main effect of visual condition 
$(\mathrm{F}(4,28)=11.8, p<0.001)$ on COM displacement. Post-hoc tests revealed significant differences in COM displacement between the visual velocity of $60 \%$ s and Dark $(p<0.01)$, $0 \% \mathrm{~s}(p<0.001)$, and $30 \% \mathrm{~s}(p<0.05)$. Significant differences were also seen between the $45 \%$ s visual velocity and Dark ( $p$ $<0.01)$ and between $45 \%$ s and $0 \%$ s $(p<0.001)$.
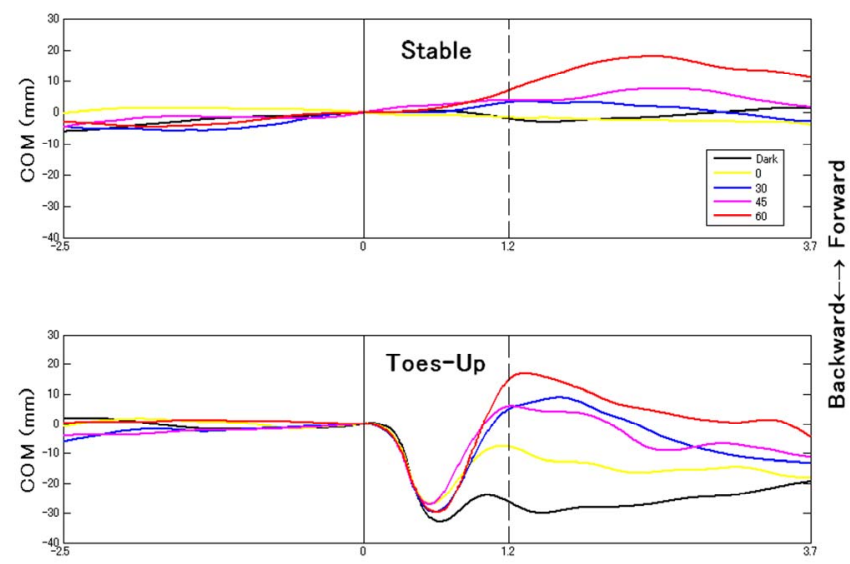

Fig. 2. COM displacements in the AP direction averaged across 3 trials of one subject in the stable and toe-up conditions. Solid vertical line indicates onset of stimulus motion. Dashed vertical line indicates the point at which 2 sec of data were analyzed. Upward traces indicate forward motion

When visual scene motion was combined with a toe-up platform (Fig. 2, bottom panel), the initial backward body sway in response to an upward tilt of the platform was followed by a corrective forward return at a mean latency of $710 \pm 70 \mathrm{msec}$ following tilt onset. There was no significant main effect of visual velocity on the latency of COM motion. There was, however, a significant main effect of visual velocity $(\mathrm{F}(4,28)=14.2, p<0.001)$ on the forward COM motion. In the Dark, the COM moved furthest from the initial position and did not fully correct to the initial vertical position during the selected timeframe. With $0 \% \mathrm{~s}$ of visual motion, there was more forward corrective motion than in the Dark, but subjects still did not return to initial vertical. In contrast, when the visual scene was moving, subjects overshot vertical by positioning their COM beyond their initial position. Magnitudes of COM motion in the Dark and at $0 \% \mathrm{~s}$ were significantly different from all other visual velocities $(p<0.001)$.

\section{B. Head angular displacement relative to the gravitational vertical}

The presence of a stable versus a toe-up platform did not significantly affect head displacement. When the platform was stable, visual motion induced an upward pitch response of the head in the same direction as the scene (Fig. 3, top panel). With increasing velocity of the visual scene, the magnitude of head angular displacement in the upward direction also increased. This increasing head angular displacement exhibited a significant change from approximately 0 deg with $0 \% \mathrm{~s}$ of visual motion to approximately $1.5 \mathrm{deg}$ with $60 \% \mathrm{~s}$ of visual motion $(\mathrm{F}(4,28)$ $=5.8, p<0.01)$. Post-hoc tests confirmed that head angular displacements with a visual scene velocity of $60 \%$ were significantly greater than those with a Dark $(p<0.05)$ or $0 \% \mathrm{~s}$ visual scene $(p<0.05)$.
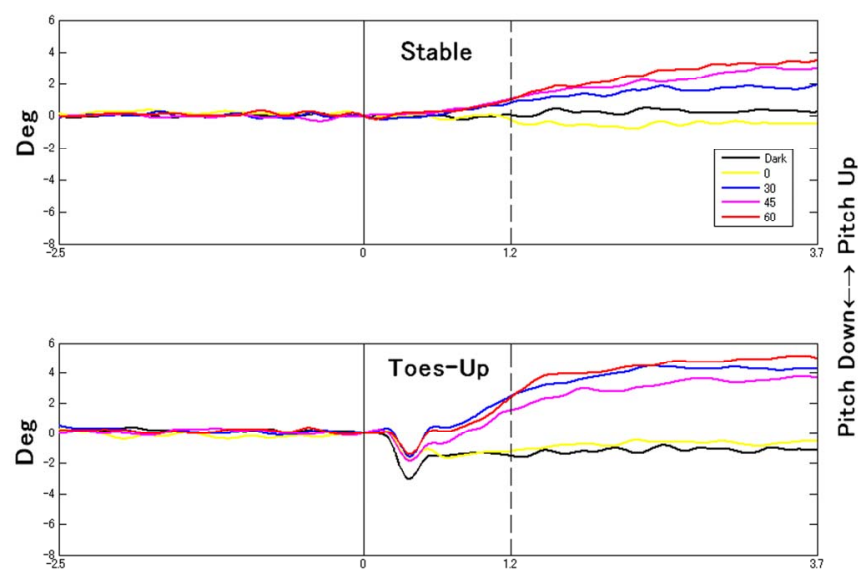

Fig. 3. Head angular displacement relative to gravitational vertical in the AP direction averaged across 3 trials of one subject in both the stable and toeup conditions. Solid vertical line indicates onset of stimulus motion. Dashed vertical line indicates the point at which $2 \mathrm{sec}$ of data were analyzed. Upward traces indicate forward motion.

When the visual scene motion was combined with a toeup platform, a transient pitch-down head response occurred. This was followed by upward pitch of the head at a latency of $570 \pm 80 \mathrm{msec}$ after stimulus onset. There was no significant effect of visual velocity on response latency. In the Dark and $0 \% \mathrm{~s}$ tasks, the head did not fully return to its initial vertical position. When the visual scene was moving, however, upward pitch motion of the head overshot the initial head position (Fig. 3, bottom panel). A significant main effect of visual velocity on head angular displacement in space was observed $(\mathrm{F}(4,28)=2.8, p<0.05)$ with no significant differences across individual velocities.

\section{Ankle angular displacement}

The presence of visual motion produced an increase in dorsiflexion motion at the ankle even with a stable platform (Fig. 4, top panel). A significant main effect of visual velocity $(\mathrm{F}(4,28)=2.8, p<0.05)$ on ankle angular displacement emerged, but with no significant differences between velocities. When the platform moved in toe-up, an obvious dorsiflexion of the ankle occurred with the motion of platform and reached its peak angular displacement approximately $590 \pm 60 \mathrm{msec}$ after stimulus onset (bottom, Fig. 4). There was no main effect $(\mathrm{F}(4,28)=0.855, p>0.05)$ of visual condition on this latency. A significant main effect of visual velocity $(\mathrm{F}(4,28)=5.6, p<0.01)$ on ankle angular displacement (bottom, Fig. 4) emerged, however, and posthoc tests revealed that ankle angular displacement with a $60 \%$ s visual velocity was significantly greater than with the Dark $(p<0.05)$ and $0 \% \mathrm{~s}(p<0.01)$ visual scenes. There was also a significant difference in ankle angular displacement $(p$ $<0.05$ ) with the $45 \%$ and $60 \%$ s visual velocities. 

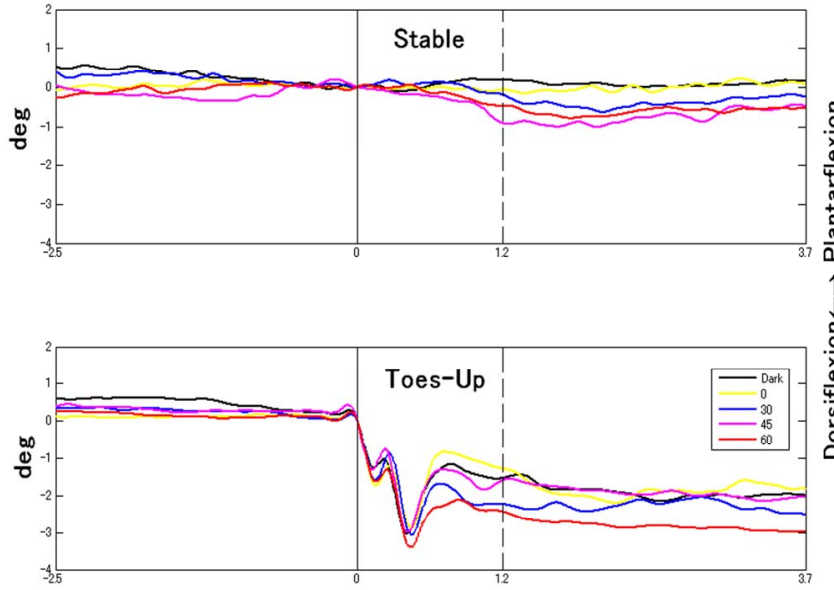

Fig. 4. Ankle angular displacement averaged across 3 trials of one subject in both the stable and toe-up conditions. Solid vertical line indicates onset of stimulus motion. Dashed vertical line indicates the point at which $2 \mathrm{sec}$ of data were analyzed. Upward traces indicate forward motion.

\section{DISCUSSION}

The goal for postural orientation is to be aligned with respect to the gravitational vertical. The ability to return to a vertical orientation, however, appears to depend very much on the ongoing sensory array. Our results clearly demonstrated that restabilization immediately following a disturbance of the base of support can be significantly affected by motion of the external world. The final orientation in space was more greatly influenced by the velocity of visual scene motion when standing on a tilted base of support from which we infer that the subjects increased their reliance on the visual field when the base of support was not earth-referenced. Given enough time, our subjects may well have returned to a vertical orientation. But their inability to recover to their initial upright position immediately following the postural disturbance could underlie the increased instability observed in patients with vestibular disorders, stroke, and elderly individuals that exhibit visual sensitivity [10], [13].

Our data also suggest that there are shared controllers for the alignment of head and ankle orientation with respect to gravitational vertical. Although head angular displacement did not differ in the stable or tilted base of support conditions, increases in the velocity of the visual scene were always accompanied by an increase in the magnitude of head angular displacement. Significant adjustments also occurred at the ankle joint across different visual conditions with both the stable condition and inclined support surfaces which would imply that the visual inputs were contributing to ankle joint stabilization as well.

\section{CONCLUSION}

Orientation information from the various senses is not always available or accurate, and the CNS has to compare incoming sensory information with an internal reference that is relevant to the postural task in order to determine the orientation of the body in space [6], [8]. This study of postural restabilization reveals that when subjects experienced concomitant disturbances of the visual and proprioceptive/vestibular systems, there was a delay in recovering their vertical orientation in space. Such a delay may have significant impact on the ability to maintain upright stance when negotiating more challenging environmental demands, particularly in individuals with damaged or aging nervous systems.

\section{REFERENCES}

[1] J. Kluzik, F. B. Horak, and R. J. Peterka, "Differences in preferred reference frames for postural orientation shown by after-effects of stance on an inclined surface," Exp Brain Res, 162, pp. 474-489, 2005.

[2] J. Kluzik, F. B. Horak, and R. J. Peterka, "Postural after-effects of stepping on an inclined surface," Neurosci Lett, 413, pp. 93-98, 2007.

[3] J. Kluzik, F. B. Horak, and R. J. Peterka, "Adaptation of postural orientation to changes in surface inclination," Exp Brain Res, 178, 117, 2007.

[4] D. E. Angelaki and J. D. Dickman, "Gravity or translation: central processing of vestibular signals to detect motion or tilt," $J$ Vestib Res, 13, pp. 245-253, 2003.

[5] L. H. Zupan and D. M. Merfeld, "An internal model of head kinematics predicts the influence of head orientation on reflexive eye movements," J Neural Eng, 2, pp. S180-197, 2005.

[6] T. Mergner and W. Becker, "A modeling approach to the human spatial orientation system," Ann N Y Acad Sci, 1004, pp. 303-315, 2003.

[7] T. Mergner and S. Glasauer, "A simple model of vestibular canalotolith signal fusion," Ann N Y Acad Sci, 871, pp. 430-434, 1999.

[8] T. Mergner, C. Maurer, and R. J. Peterka, "A multisensory posture control model of human upright stance," Prog Brain Res, 142, pp. 189-201, 2003.

[9] V. S. Gurfinkel, Yu P. Ivanenko, Yu S. Levik, and I. A. Babakova, "Kinesthetic reference for human orthograde posture," Neuroscience, 68, pp. 229-243, 1995.

[10] E. A. Keshner, J. Streepey, Y. Dhaher, and T. Hain, "Pairing virtual reality with dynamic posturography serves to differentiate between patients experiencing visual vertigo," $J$ Neuroengineering Rehabil, 4, pp. 24, 2007.

[11] E. A. Keshner, J. H. Allum, and C. R. Pfaltz, "Postural coactivation and adaptation in the sway stabilizing responses of normals and patients with bilateral vestibular deficit," Exp Brain Res, 69, pp. 7792, 1987.

[12] S. L. Whitney, P. J. Sparto, L. F. Hodges, S. V. Babu, J. M. Furman, and M. S. Redfern, "Responses to a virtual reality grocery store in persons with and without vestibular dysfunction," Cyberpsychol Behav, 9, pp. 152-156, 2006.

[13] M. Guerraz, L. Yardley, P. Bertholon, L. Pollak, P. Rudge, M. A. Gresty, and A. M. Bronstein, "Visual vertigo: symptom assessment, spatial orientation and postural control," Brain, 124, pp. 1646-1656, 2001 